\title{
A Cystic Fibrosis Respiratory Epithelial Cell Chronically Treated by Miglustat Acquires a Non-Cystic Fibrosis-Like Phenotype
}

\author{
Caroline Norez ${ }^{1}$, Fabrice Antigny ${ }^{1}$, Sabrina Noel ${ }^{1}$, Clarisse Vandebrouck ${ }^{1}$, and Frédéric Becq ${ }^{1}$ \\ ${ }^{1}$ Institut de Physiologie et Biologie Cellulaires, Université de Poitiers, Centre National de la Recherche Scientifique, Poitiers, France
}

\begin{abstract}
Cystic fibrosis (CF) is a fatal, autosomal and recessive genetic disease that is mainly due to inactivating mutations in the chloride channel CF transmembrane conductance regulator (CFTR). Sodium hyperabsorption by the airways, profound lung inflammation, and dysregulation of calcium homeostasis, are presumably causally related to loss of CFTR-dependent chloride function in patients with CF. Miglustat (N-butyldeoxynojirimycin, Zavesca), an inhibitor of the $\alpha-1,2$ glucosidase, has been proposed for clinical use in CF because of its effect as a corrector of the defective trafficking of F508del-CFTR. In the present study, we show that daily treatment for $\mathbf{2}$ months with low concentrations of miglustat on the human $C F$ nasal epithelial cell line, JME/CF15 (F508del/F508del-CFTR), results in progressive, stable, reversible, and sustained correction of F508del-CFTR trafficking, down-regulation of sodium hyperabsorption, and regulation of the calcium homeostasis. In conclusion, we provide here the first evidence that a respiratory CF cell can acquire a non-CF-like phenotype when chronically treated with low concentrations of a pharmacological drug.
\end{abstract}

Keywords: F508del-cystic fibrosis transmembrane conductance regulator; epithelial $\mathrm{Na}^{+}$channel; calcium homeostasis; sodium hyperabsorption; miglustat

Cystic fibrosis (CF), also called mucoviscidosis, is a lethal, autosomal, recessive genetic polyexocrinopathy, arising from mutations in the gene encoding the CF transmembrane conductance regulator (CFTR) protein. CFTR functions as a cyclic adenosine monophosphate (cAMP)-activated and ATP-gated $\mathrm{Cl}^{-}$channel, which is expressed in the apical membrane of a wide variety of epithelial cell types (1); however, the major morbidity and mortality associated with $\mathrm{CF}$ relate to pulmonary disease. Although over 1,000 different disease-associated mutations that result in defective or dysfunctional CFTR channels have been described to date, approximately $70 \%$ of patients with $\mathrm{CF}$ are homozygous for a single mutation, the F508delCFTR mutation, with roughly $90 \%$ of all patients possessing at least one F508del-CFTR allele (2). Trafficking of the F508delCFTR protein from the endoplasmic reticulum (ER) to the plasma membrane is extremely inefficient (2) and compromises chloride channel function. Moreover, the protein has a much shorter half-life in the plasma membrane than the wild-type CFTR (3). Although the gating of the channel is affected by the F508 deletion, the mutant protein retains some functionality as a $\mathrm{Cl}^{-}$channel (4). For this reason, therapeutic efforts have been aimed at overcoming the trafficking defect to increase the amount of F508del-CFTR present at the cell membrane (5-7).

(Received in original form July 25, 2008 and in final form November 5, 2008)

This work was supported by grants from Vaincre la Mucoviscidose (VLM), MucoVie66 and ABCF2. C.N. and F.A. were supported by student fellowships from VLM, and S.N. was supported by a student fellowship from MucoVie66.

Correspondence and requests for reprints should be addressed to Frédéric Becq, IPBC, Université de Poitiers, 40 Avenue du Recteur Pineau, 86022 Poitiers, France. E-mail: frederic.becq@univ-poitiers.fr

Am J Respir Cell Mol Biol Vol 41. pp 217-225, 2009

Originally Published in Press as DOI: 10.1165/rcmb.2008-02850C on January 8, 2009

Internet address: www.atsjournals.org

\section{CLINICAL RELEVANCE}

We show that a respiratory cystic fibrosis (CF) cell can acquire a non-CF-like phenotype when chronically treated with low-concentration miglustat. This drug is thus attractive as a potential pharmacologic therapy for patients with CF who have at least one F508del-CF transmembrane conductance regulator allele.

However, the pathophysiology of CF is complex, and not neatly ascribed to loss of the CFTR $\mathrm{Cl}^{-}$channel function. Other cellular processes, seemingly unrelated to $\mathrm{Cl}^{-}$conductance, such as $\mathrm{Na}^{+}$absorption, $\mathrm{Ca}^{2+}$ homeostasis, and inflammatory response, are also deeply disturbed in CF cells (8).

New therapeutics aimed at improving mutant CFTR functions, also known as "protein repair therapy," are hoped and predicted to replace some of the currently used therapy, and to reduce the financial burden for patients with $\mathrm{CF}$, while improving the quality of life as well as life expectancy. Recently, we investigated the effects of a high concentration $(100 \mu \mathrm{M})$ of miglustat on several CF characteristics, and demonstrated, after short-term (2-4 hours) treatment of CF cells, a partial rescue of the defective F508del-CFTR trafficking and function (9), an improvement in the abnormal $\mathrm{Ca}^{2+}$ homeostasis (10), and a down-regulation of epithelial $\mathrm{Na}^{+}$channel (ENaC)-dependent $\mathrm{Na}^{+}$hyperabsorption (11). Miglustat is now evaluated in patients with $\mathrm{CF}$ within a pilot phase 2 a clinical trial (http://clinicaltrials. gov/). We suggested that the mechanism by which miglustat corrects the defective F508del-CFTR trafficking is correlated with a disturbance of the ER quality control system in CF cells. In support of this suggestion, miglustat is an $\alpha$-1,2-glucosidase inhibitor preventing the interaction between F508del-CFTR and calnexin in the ER (9).

Other small molecules, such as curcuminoids (12-14), 4phenylbutyrate (4-PBA, buphenyl) $(15,16)$, and CFTRcor-325 (17), are also potential candidates to rescue F508del-CFTR function. Most of these small molecules act after short-term incubation (2-24 hours, depending on studies and laboratories) of CF cells, and little if any information is available concerning their long-term effect. In this study, we address the important issue of the concentration and time dependence of action of one corrector, miglustat, on human $\mathrm{CF}$ nasal epithelial cell line, JME/CF15 (F508del/F508del-CFTR). We examine ionic transports in CF cells cultured in the presence of the drug, and report here that daily treatment for 2 months with low-concentration miglustat results in progressive, stable, reversible, and sustained correction of F508del-CFTR trafficking, down-regulation of sodium hyperabsorption, and regulation of calcium homeostasis. In conclusion, we provide here the first evidence that a respiratory $\mathrm{CF}$ cell can acquire a non-CF-like phenotype when chronically treated with low concentrations of a pharmacological drug. 


\section{MATERIALS AND METHODS}

\section{Cell Culture}

For this study, we used the human nasal airway epithelial cell line, JME/CF15, derived from a patient with CF homozygous for the F508 del mutation (18). The details of the generation, characterization, and routine propagation have been described elsewhere $(14,18)$. For chronic treatment, cells were routinely replenished every 24 hours with fresh medium containing $3 \mu \mathrm{M}$ miglustat. It is important to note that miglustat is a water-soluble compound.

\section{Immunoprecipitation and Western Blot Analysis}

For immunoprecipitation, cell lysates were incubated overnight at $4{ }^{\circ} \mathrm{C}$ with $2 \mu \mathrm{g}$ of CFTR antibody (monoclonal anti-human CFTR antibody, $\mathrm{IgG}_{2 \mathrm{a}}$ clone 24-1; R\&D Systems, Minneapolis, MN). To precipitate immune complexes, incubation with $3 \mu \mathrm{g}$ of protein G-sepharose (Amersham Pharmacia Biotech, Orsay, France) was conducted for 1 hour at $4^{\circ} \mathrm{C}$. Samples were separated on SDS-PAGE (7\% polyacrylamide gel) and analyzed by Western blot. Immunoblots were probed overnight at $4^{\circ} \mathrm{C}$ with primary anti-calnexin antibody $(2 \mu \mathrm{l} / \mathrm{ml}$, polyclonal rabbit anti-calnexin antibody, SPA-860; Stressgen, Strasbourg, France). The protein levels were determined as densitometry of band intensity and expressed as percentage of untreated cells. Other details can be found elsewhere (14).

\section{lodide Efflux}

The cAMP-dependent CFTR $\mathrm{Cl}^{-}$channel activity was assessed by measuring the rate of iodide $\left({ }^{125} \mathrm{I}\right)$ efflux from cells, as previously described (14). Curves were constructed by plotting the rate of ${ }^{125} \mathrm{I}$ versus time. All comparisons were based on maximal values for the time-dependent rates $\left(\mathrm{k}=\right.$ peak rates, $\left.\mathrm{min}^{-1}\right)$, excluding the points used to establish the baseline $\left(\mathrm{k}_{\text {peak }}-\mathrm{k}_{\mathrm{basal}}, \min ^{-1}\right)(14,19)$.

\section{Patch-Clamp Experiments}

Perforated whole-cell patch-clamp experiments were performed on CF15 cells at room temperature. Importantly, in these experiments, the composition of intrapipette solution allowed the recording of $\mathrm{Cl}^{-}$ currents only, and the simultaneous presence in the bath solution of calixarene and DIDS (4,4'-diiosothiacyanatostilbene-2,2'-disulfonate) prevented activation of non-CFTR $\mathrm{Cl}^{-}$currents, as previously described (9). Current-voltage relationships were built by clamping the membrane potential to $-40 \mathrm{mV}$ and by pulses from -80 to $+80 \mathrm{mV}$ (20 mV increments) (other details can be found in Ref. 9).

\section{Recording Calcium Signals}

In CF cells loaded with $3 \mu \mathrm{M}$ Fluo-4 acetoxymethyl (AM)ester (FluoProbes, Montluçon, France), $\mathrm{Ca}^{2+}$ activity was recorded by confocal laser scanning microscopy using a Bio-Rad MRC 1,024 (Hemel Hempstead, UK) equipped with $15 \mathrm{~mW} \mathrm{Ar} / \mathrm{Kr}$ gas laser as previously described $(10,14)$.

\section{Single-Cell Measurement of CFTR and ENaC Activities by Fluorescence Imaging}

Ion channel functions were assessed by single-cell fluorescence imaging using the potential-sensitive probe, bis-(1,3-diethylthiobarbituric acid)trimethine oxonol (DiSBAC 2 [3]; Molecular Probes, Eugene, OR), as previously reported (20), but with minor changes: fluorescence intensity was recorded by confocal laser scanning microscopy using BioRad MRC 1,024 equipped with $15 \mathrm{~mW} \mathrm{Ar/Kr}$ gas laser. Maximal resolution was obtained with an Olympus plan apo $\times 60$ oil, 1.4 numerical aperture, objective lens. Fluorescence signal collection was performed through the control software, Lasersharp 3.2 (Hemel). The resolution time was 30 seconds. Bis-oxonol slowly distributes across biological membrane, according to the membrane potential, and binds to hydrophobic cell components; because the quantum yield of the dye increases impressively upon binding, the fluorescence of cells incubated in a medium containing bis-oxonol increases upon depolarization and, conversely, decreases with hyperpolarization (21). In our experiments, CFTR-dependent current was stimulated by application of forskolin (Fsk) plus genistein (Gst), inducing a depolarization characterized by an increase in fluorescence. On the contrary, ENaC-dependent current was inhibited by application of amiloride, inducing a hyperpolarization characterized by a decrease in fluorescence. Results are presented as transformed data to obtain the percent signal variation $(\mathrm{Fx})$ relative to the time of addition of the stimulus, according to the equation: $\mathrm{Fx}=$ $\left(\left[F_{t}-F_{0}\right] / F_{0}\right] \times 100$, where $F_{t}$ and $F_{0}$ are the fluorescent values at time $t$ and at the time of addition of stimulus, respectively. For histogram representation, the values correspond to the level of stable variation of fluorescence induced by each drug (for other details, see Ref. 20).

\section{Statistical Analysis}

Results are expressed as mean $( \pm$ SEM) of $n$ or $N$ observations ( $n$ represents the number of isolated cells, and $N$ the number of cell populations tested). Sets of data were compared with Student's $t$ test. Differences were considered statistically significant with a $P$ value less than 0.05. All statistical tests were performed using GraphPad Prism version 4.0 for Windows (Graphpad Software, La Jolla, CA) and Origin version 5.0.

\section{Chemicals and Reagents}

Miglustat was purchased from Toronto Research Chemicals (Toronto, Canada). Fsk and Gst were from LC Laboratories (Woburn, MA).

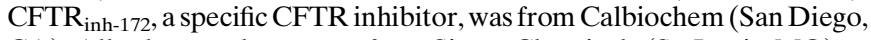
CA). All other products were from Sigma Chemicals (St. Louis, MO).

\section{RESULTS}

\section{The Correcting Effect of Miglustat Depends on the Concentration and Duration of Incubation}

Recently, we demonstrated that a short incubation ( 2 hours) of a high concentration $(100 \mu \mathrm{M})$ of miglustat was sufficient and necessary to partially rescue the defective F508del-CFTR function (9). In the first part of the present study, we analyzed the impact of modifying the concentration and duration of miglustat treatment on this rescue. In our experiments, to stimulate the chloride channel function of F508del-CFTR, we used a mixture composed of Fsk (an adenylate cyclase activator raising the cellular cAMP level) and Gst (the isoflavone, Gst, is a direct activator of CFTR) (14). We increased the duration of incubation of CF cells from 1 to 14 hours in the presence of miglustat added at a final concentration of $100 \mu \mathrm{M}$ in the culture medium at $37^{\circ} \mathrm{C}$. Figure $1 \mathrm{~A}$ illustrates the effect of 2- or 6 -hour treatment. Note that Fsk-Gst stimulated an approximately twofold higher CFTR-dependent iodide efflux at 6 hours $\left(\mathrm{k}_{\text {peak }}-\mathrm{k}_{\text {basal }}=0.123 \pm 0.008 \min ^{-1} ; n=4\right)$ than at 2 hours $\left(\mathrm{k}_{\text {peak }}-\mathrm{k}_{\text {basal }}=0.068 \pm 0.008 \min ^{-1} ; n=4\right)$. We observed that the correcting effect was time dependent, with a maximal effect obtained after 4-6 hours of treatment, and then progressively declined with a total loss of effect after 14 hours (Figure 1B). Thus, the rescue of F508del-CFTR function by miglustat is dependent on the duration of cell incubation.

We then asked whether increasing the time of incubation of cells with the drug would have an impact on the half maximal effective concentration values $\left(\mathrm{EC}_{50}\right)$. We determined $\mathrm{EC}_{50}$ with CF15 cells incubated for 2, 4, 6, 8, 10, 12, and 14 hours in the presence of increasing concentrations of miglustat. The F508del-CFTR channel activity was determined for each time and for each concentration with the iodide efflux method. We plotted the rate of efflux (noted as $\mathrm{k}_{\text {peak }}-\mathrm{k}_{\text {basal }}, \mathrm{min}^{-1}$ ) as a function of the concentration of miglustat (Figure 1C) to calculate $\mathrm{EC}_{50}$. Interestingly, we observed a decrease in $\mathrm{EC}_{50}$ values as a function of the duration of the treatment, from $112( \pm 1.2) \mu \mathrm{M}$ for 2-hour treatment to $40( \pm 1.0) \mu \mathrm{M}, 15$ $( \pm 1.1) \mu \mathrm{M}, 7.4( \pm 1.2) \mu \mathrm{M}, 1.2( \pm 1.2) \mu \mathrm{M}$, and $1.2( \pm 1.2) \mu \mathrm{M}$ for $4,6,8,10$, and 12-hour treatments, respectively. Note that, after the 14-hour treatment, no rescue was measured at any concentration of the drug. Collectively, these data demonstrate 

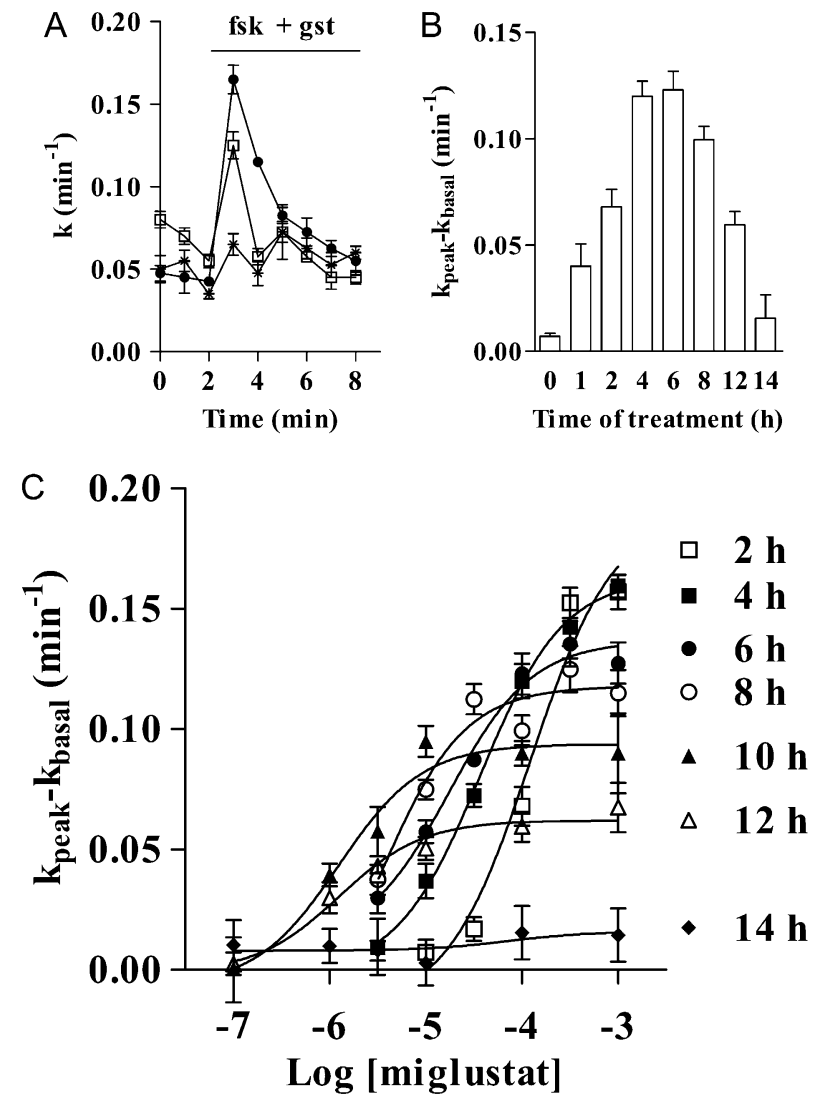

Figure 1. Time- and concentration-dependent correction by miglustat. F508del-cystic fibrosis (CF) transmembrane conductance regulator (CFTR) activity was assayed with the iodide efflux technique in the presence of forskolin (Fsk; $10 \mu \mathrm{M}$ ) plus genistein (Gst; $30 \mu \mathrm{M}$ ). (A) Examples of iodide efflux curves as function of time on CF15 cells cultured at $37^{\circ} \mathrm{C}$ (asterisks), $37^{\circ} \mathrm{C}$ plus $100 \mu \mathrm{M}$ miglustat for 2 hours (open squares) and $37^{\circ} \mathrm{C}$ plus $100 \mu \mathrm{M}$ miglustat for 6 hours (closed circles). (B) Time dependence of F508del-CFTR trafficking correction induced by miglustat $(100 \mu \mathrm{M})$. (C) Concentration-response curves with CF15 cells incubated with miglustat over the period indicated on the right of the graph. $(A-C)$ Results are expressed as means ( \pm SEM) of four experiments.

correction of endogenous F508del-CFTR $\mathrm{Cl}^{-}$channel function with miglustat in a time- and concentration-dependent manner. With several hours of treatment, low effective concentrations can be achieved, whereas, with short-term treatment, high effective concentrations are necessary.

\section{Correlation between the F508del-CFTR Trafficking Rescue and the Prevention of the Calnexin/ F508del-CFTR Interaction}

Previously, we suggested that the rescue of F508del-CFTR abnormal function after 2-hour treatment with miglustat was due to prevention of the calnexin/F508del-CFTR interaction in the ER (9). To investigate whether this molecular explanation could be confirmed as a function of time, we performed coimmunoprecipitation studies on CF cells treated for 1-12 hours with miglustat. Two types of control have been done using CF15 lysate and mouse IgG (Figure 2A, lanes $a$ and $b$ ). As expected (9), after immunoprecipitation with CFTR antibody, anti-calnexin Western blot showed a decrease of the calnexin band intensity by miglustat treatment $(75 \%$ inhibition; Figure $2 \mathrm{~A}$, lane $e$ ) after 2 hours of treatment. Furthermore, we found that miglustat progressively prevents F508del-CFTR/calnexin interaction (Figure 2A, lanes $d-g$ ) as compared with $\mathrm{CF} 15$ at $37^{\circ} \mathrm{C}$ (Figure $2 \mathrm{~A}$, lane $\mathrm{c}$ ), but we also observed a lack of effect of miglustat after 8 hours (Figure 2A, lanes $h$ and $i$ ). To verify that the effect of miglustat was not due to a direct effect on the production of calnexin itself, we performed Western blot with the anti-calnexin antibody using lysates of CF15 incubated after the same experimental conditions as above. As already reported for a 2-hour miglustat treatment (9), none of these treatments affect calnexin production (data not shown). Figure $2 \mathrm{~B}$ presents quantification from three separate experiments as a function of the duration of incubation of F508del-CFTR/calnexin interaction together with the rescue of F508del-CFTR iodide efflux to quantify the channel function for the same time of incubation. These experiments demonstrated a good correlation between the prevention of the F508del-CFTR/calnexin interaction and the restoration of F508del-CFTR function. Moreover, inspection of the two curves revealed a dynamic process; the time period for which the interaction between calnexin and F508delCFTR protein is the weakest corresponds to the maximal rescue of channel function. In contrast, when the interaction between the two proteins was not perturbated (by miglustat), we did not observe rescue of F508del-CFTR function.

\section{Normalization of Calcium Response and Rescue of F508del-CFTR}

Similarly, in a second series of experiments, we wished to evaluate the time dependence of miglustat-induced $\mathrm{Ca}^{2+}$ normalization that we prviously observed (10) after 2-hour treatment (Figure 3A). Figure 3B presents the quantification of the area under the curve for the $\mathrm{Ca}^{2+}$ response (examples are given Figure 3A) measured regularly during a 24-hour period of treatment with miglustat. After the addition of histamine, we recorded a biphasic $\mathrm{Ca}^{2+}$ response (Figure 3A), which was decreased when cells were treated for 2-12 hours with miglustat (Figures $3 \mathrm{~A}$ and $3 \mathrm{~B}$ ). This down-regulation was not only dependent on the time of incubation, because, after 16 hours of treatment, the $\mathrm{Ca}^{2+}$ response returned to the control level (Figure 3B), but was also in phase with the rescue of F508delCFTR function (Figure 3A, black squares). Interestingly, the temporal alteration in the calcium response of CF15 cells appears to be slightly delayed compared with the effects seen on iodide efflux. Indeed, $\mathrm{Ca}^{2+}$ responses only fully revert toward the CF phenotype after roughly 16 hours, whereas the iodide efflux takes approximately 14 hours. We interpret these data as an argument for normalization of the $\mathrm{Ca}^{2+}$ homeostasis being a consequence of the F508del-CFTR correction. As they are two different parameters, the turnover of each parameter is mutually independent.

\section{Effect of the Refreshment of Miglustat on the Rescue of F508del-CFTR Function}

To further explore the time dependence of the miglustat correction, in a separate series of experiments we incubated CF15 cells for 2 hours at $37^{\circ} \mathrm{C}$ with $100 \mu \mathrm{M}$ miglustat, then washed out the drug and analyzed the Fsk/Gst iodide efflux response every 2 hours for 12 hours. The rescue of F508del-CFTR function was maximal during the first 4 hours after compound washout, and then progressively declined between 6 and 12 hours (Figure 4A). Similar experiments were conducted to measure the $\mathrm{Ca}^{2+}$ mobilization after miglustat treatment, and again showed a normalized $\mathrm{Ca}^{2+}$ response during the first 6 hours and then a progressive loss of effect for longer washout (data not shown). Importantly, when we incubated CF15 cells for 12 hours, renewing the drug after 6 hours, we found a significantly increased Fsk/Gst response $(P<0.001)$ compared with cells treated for 12 hours without renewing the drug (Figure 4B). 
A

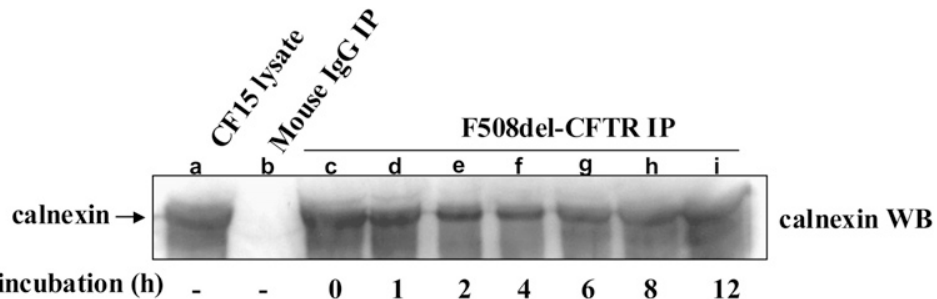

Figure 2. Miglustat prevents interaction of F508del-CFTR with calnexin in a time-dependent manner. $(A)$ Coimmunoprecipitation of CFTR with calnexin on CF15 cells $\begin{array}{llllllllll}\text { Time of incubation (h) } & - & - & 0 & 1 & 2 & 4 & 6 & 8 & 12\end{array}$ treated with miglustat $100 \mu \mathrm{M}$ over the period indicated under the gel (lanes $c-i)$. CF15 lysate (lane $a$ ) and nonimmune mouse IgG (lane $b$ ) were used as positive and negative controls. $(B)$ Correlation between quantifications of F508del-CFTR activity rescue (closed squares; $n=4$ ) and of the F508del-CFTR/calnexin interaction (open squares; $n=3$ ) as a function of time of miglustat incubation. F508del-CFTR activity was assayed with iodide efflux experiments in the presence of Fsk $(10 \mu \mathrm{M})$ plus Gst $(30 \mu \mathrm{M})$ on CF15 cells incubated with miglustat $100 \mu \mathrm{M}$ over the period indicated.

Next, we examined the effect of low-concentration miglustat $(3 \mu \mathrm{M})$ during 6 days of treatment with daily renewal of the drug. Figure 4C shows a lack of miglustat effect after only 1 day of treatment (as expected from Figure 1C), but then a progressive rescue of F508del-CFTR function beginning after 2 days of treatment, being maximal and then stable after 4 days of treatment. This level of rescue with $3 \mu \mathrm{M}$ miglustat during 4 days is somewhat similar to that obtained after short-term (4 hours) and high concentration $(100 \mu \mathrm{M})$ of the drug (compare
Figures $1 \mathrm{~A}$ and $4 \mathrm{C}$ ). We next performed electrophysiological experiments to directly address the F508del-CFTR $\mathrm{Cl}^{-}$channel function in $\mathrm{CF}$ epithelial cells challenged with miglustat. We used the perforated mode of the patch-clamp technique in three different experimental situations (i.e., with CF15 cells cultured at $37^{\circ} \mathrm{C}$ ) (Figure $5 \mathrm{~A}$ ) or with $\mathrm{CF} 15$ cells cultured at $37^{\circ} \mathrm{C}$ in the presence of $3 \mu \mathrm{M}$ miglustat for 8 hours (Figure $5 \mathrm{~B}$ ), or 6 days with a refreshment of miglustat every 24 hours (Figure 5C). In these experiments, the composition of intrapipette solution
A
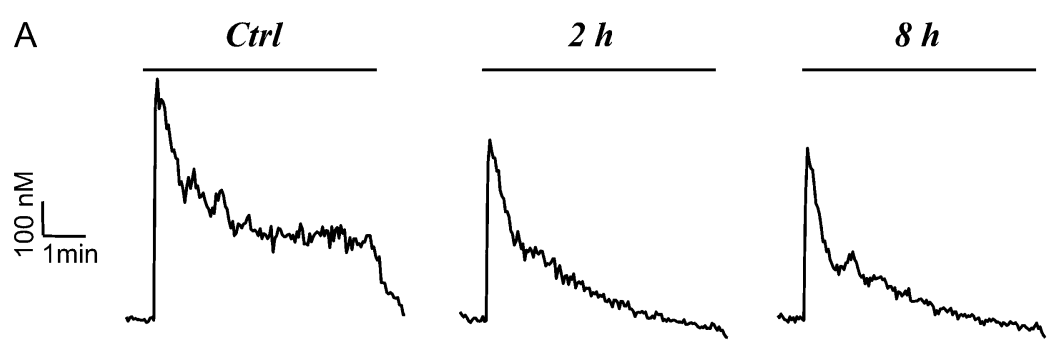

B

$B$
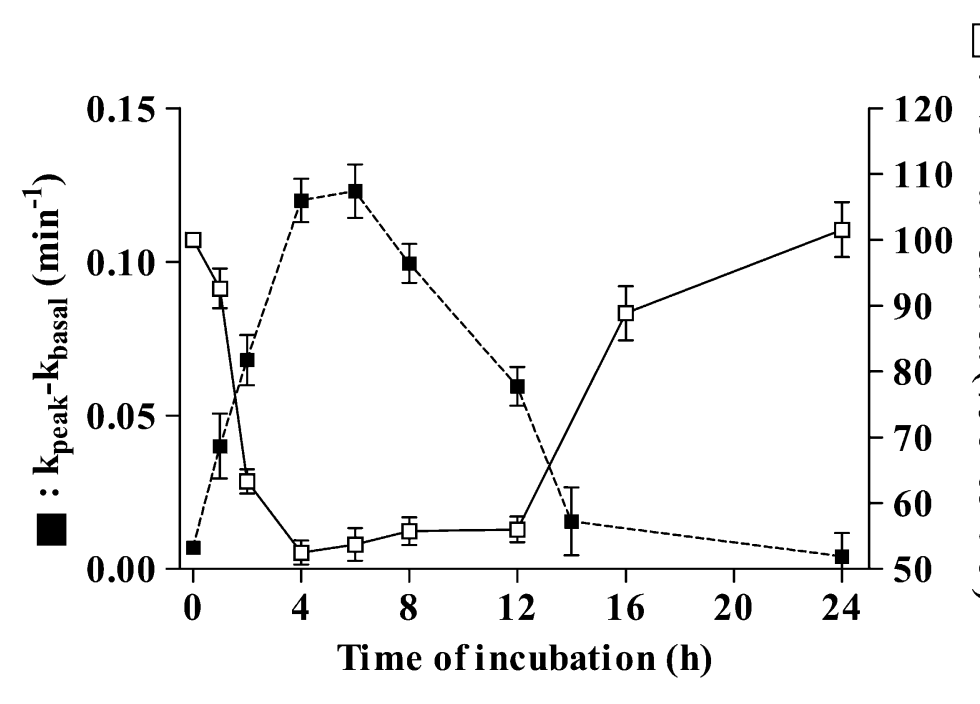

\section{$24 h$}

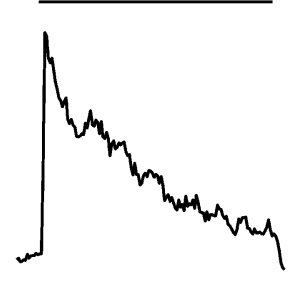

Figure 3. Normalization of $\mathrm{Ca}^{2+}$ response by miglustat is time dependent. (A) Typical traces of $\mathrm{Ca}^{2+}$ mobilization induced by histamine $(100 \mu \mathrm{M}$, 5 minutes; black line on the top of the trace) in untreated CF15 cells (Ctrl) or in CF15 cells treated with $100 \mu \mathrm{M}$ miglustat during 2, 8 or 24 hours. (B) Relationship between $\mathrm{Ca}^{2+}$ mobilization variations (open squares; $35<$ $n<85$ ) and F508del-CFTR rescue (closed squares; $n=4$ ) as a function of time of miglustat incubation. Quantification of F508del-CFTR activity was assayed with iodide efflux experiments in the presence of Fsk $(10 \mu \mathrm{M})$ plus Gst (30 $\mu \mathrm{M})$ on CF15 cells incubated with $100 \mu \mathrm{M}$ miglustat over the period indicated. 


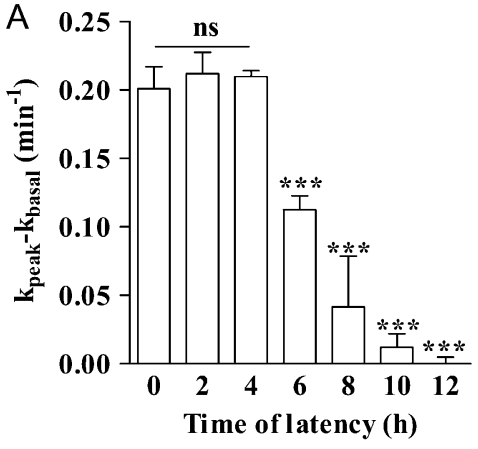

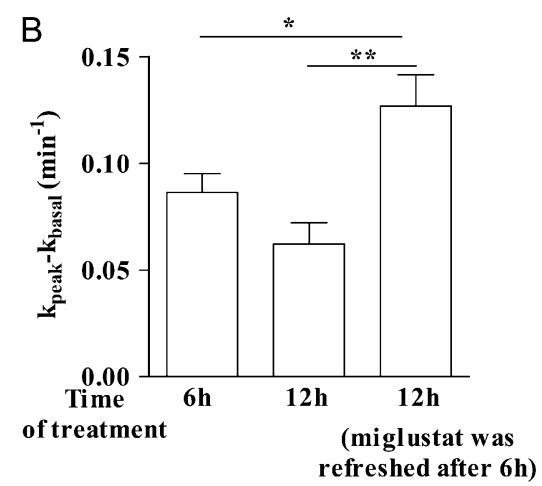

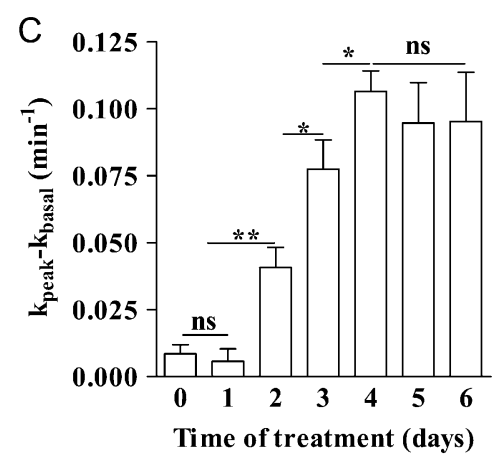

Figure 4. Persistence of the F508del-CFTR correction by miglustat. F508del-CFTR activity was assayed with iodide efflux experiments in presence of Fsk $(10 \mu \mathrm{M})$ plus Gst $(30 \mu \mathrm{M})$. (A) CF15 cells were treated 2 hours with $100 \mu \mathrm{M}$ miglustat, rinsed, and then F508del-CFTR activity was assayed at different times after washout. (B) Recovery of the F508del-CFTR activity after removing treatment. CF15 cells were treated with $100 \mu M$ miglustat during 6 hours, 12 hours without media change, or 12 hours refreshing miglustat after 6 hours. (C) Persistence of the F508del-CFTR correction by miglustat. CF15 cells were treated with $3 \mu \mathrm{M}$ miglustat during 6 days refreshing miglustat every 24 hours. $(A-C) n=4$ for each experiment; $* * * P<$ $0.001 ;{ }^{* *} P<0.01 ;{ }^{*} P<0.05 ;$ ns, no significant difference.

allowed the recording of $\mathrm{Cl}^{-}$currents only. The simultaneous presence in the bath solution of calixarene and DIDS prevented activation of non-CFTR $\mathrm{Cl}^{-}$currents. For each protocol, $\mathrm{Cl}^{-}$ currents were first recorded in resting cells (Figures 5A-5C, top traces). CF15 cells were then stimulated by the application of the mixture of $10 \mu \mathrm{M}$ Fsk plus $30 \mu \mathrm{M}$ Gst. We recorded a linear, non-voltage-dependent $\mathrm{Cl}^{-}$current for preparations either treated for 8 hours (Figure 5B, middle traces) or incubated for 6 days with $3 \mu \mathrm{M}$ miglustat (Figure 5C, middle traces). Untreated CF15 cells failed to respond to Fsk/Gst (Figure 5A, middle traces), indicating a lack of functional F508del-CFTR. The rescued F508del-CFTR $\mathrm{Cl}^{-}$current was fully inhibited by the bath application of glibenclamide (Figure 5B, bottom traces) or $\mathrm{CFTR}_{\text {inh-172 }}$ (Figure $5 \mathrm{C}$, bottom traces). The corresponding curves for current densities as a function of voltage are presented below each panel. These experiments showed, first, that CF cells treated for 6 days with miglustat are rescued and, second, that the amplitude of the current densities recorded on cells treated for 8 hours or 6 days are similar.

\section{Effect of the Chronic Treatment of CF Cells with Miglustat}

Finally, we explored long-term treatment of CF cells with lowconcentration miglustat. We incubated $\mathrm{CF}$ cells with $3 \mu \mathrm{M}$ miglustat and refreshed the medium every 24 hours for 58 days. Because CF epithelial cells in airway, both CFTR-dependent chloride and amiloride-sensitive sodium transports (mediated by $\mathrm{ENaC}$ ) are abnormal (8), we wished to evaluate the activities of both ionic channels in a single cell challenged with miglustat over the 58-day period. To that end, we measured ionic transport regularly by single-cell fluorescence imaging using the membrane potential-sensitive fluorescent probe, $\operatorname{DiSBAC}_{2}(3)$. As shown in Figure 6A (left panel), a sharp decrease of fluorescence, corresponding to a membrane hyperpolarization (see Materials AND Methods), was recorded in untreated CF15 cells after addition of amiloride $(100 \mu \mathrm{M})$, but no variation of the fluorescence was recorded after addition of the CFTR activators, Fsk plus Gst. These results confirm the absence of functional F508del-CFTR in untreated CF15 cells, and the presence of a significant amiloride-sensitive $\mathrm{Na}^{+}$current (11). In contrast, after 58 days in the presence of miglustat (Figure 6A, right panel), CF15 cells did not respond to amiloride, but displayed a significant CFTR-sensitive current, characterized on the trace by a fluorescence increase after addition of Fsk plus Gsk. The decrease in the fluorescence level observed after addition of CFTR $_{\text {inh-172 }}$ further confirmed the identity of CFTR. Remarkably, intermediary profiles were observed (e.g., for the 2-day treatment) (Figure 6A, middle panel), which yielded recording of moderate $\mathrm{ENaC}$ and F508del-CFTR activities.

In Figure 6B, we have plotted the value of fluorescence measured between Days 2 and 58 of treatment (as described in Materials and Methods). When CF15 cells were cultured in the presence of $3 \mu \mathrm{M}$ miglustat for up to 4 days, there was a statistically significant decrease in the amiloride-sensitive $\mathrm{ENaC}$ current, concomitant with the rescue of F508del-CFTRdependent current. These effects were augmented with increasing time of miglustat exposure up to Day 4 of treatment. Then, after Day 4, the regulation of these two currents remained maximal and similar to the level obtained after short-term (2-hour) and high-concentration $(100 \mu \mathrm{M})$ incubation of the drug (Figure $6 \mathrm{~B}$, last bar on the right). We also investigated the long-term effect of miglustat exposure on the $\mathrm{Ca}^{2+}$ response of CF cells, and measured similar progressive and maintained normalization (data not shown).

Finally, we wished to know whether the long-term rescue could be reversible. To explore this possibility, we used cells maintained for 54 days in $3 \mu \mathrm{M}$ miglustat, and then washed out the drug and analyzed the amiloride and Fsk/Gst fluorescence changes every day for 4 days. Figure 7A (left trace) illustrates the response obtained after 54 days (used as control), or after 2 or 4 days in the absence of the drug. In Figure $7 \mathrm{~B}$, we have plotted the values of fluorescence measured between Days 1 and 4 of washout, which shows the progressive reversibility of the response (loss of F508del-CFTR rescue and increased amiloride-sensitive response) to the level measured in untreated CF cells (Figure 6A, ctrl). Therefore, the long-term rescue of $\mathrm{CF}$ cells toward a non-CF-like phenotype is reversible.

\section{DISCUSSION}

Our aim in the present study was to explore the concentration and time dependence of miglustat-induced correction of ionic transport in the human respiratory $\mathrm{CF}$ epithelial cell line, CF15 (F508 del/F508del-CFTR). The most salient result is the demonstration that a daily treatment for 2 months with lowconcentration miglustat resulted in progressive, stable, reversible, and sustained correction of the F508del-CFTR-deficient trafficking. Then, by investigating different biological and cellular aspects of $\mathrm{CF}$, such as $\mathrm{Na}^{+}$hyperabsorption and dysregulation of 

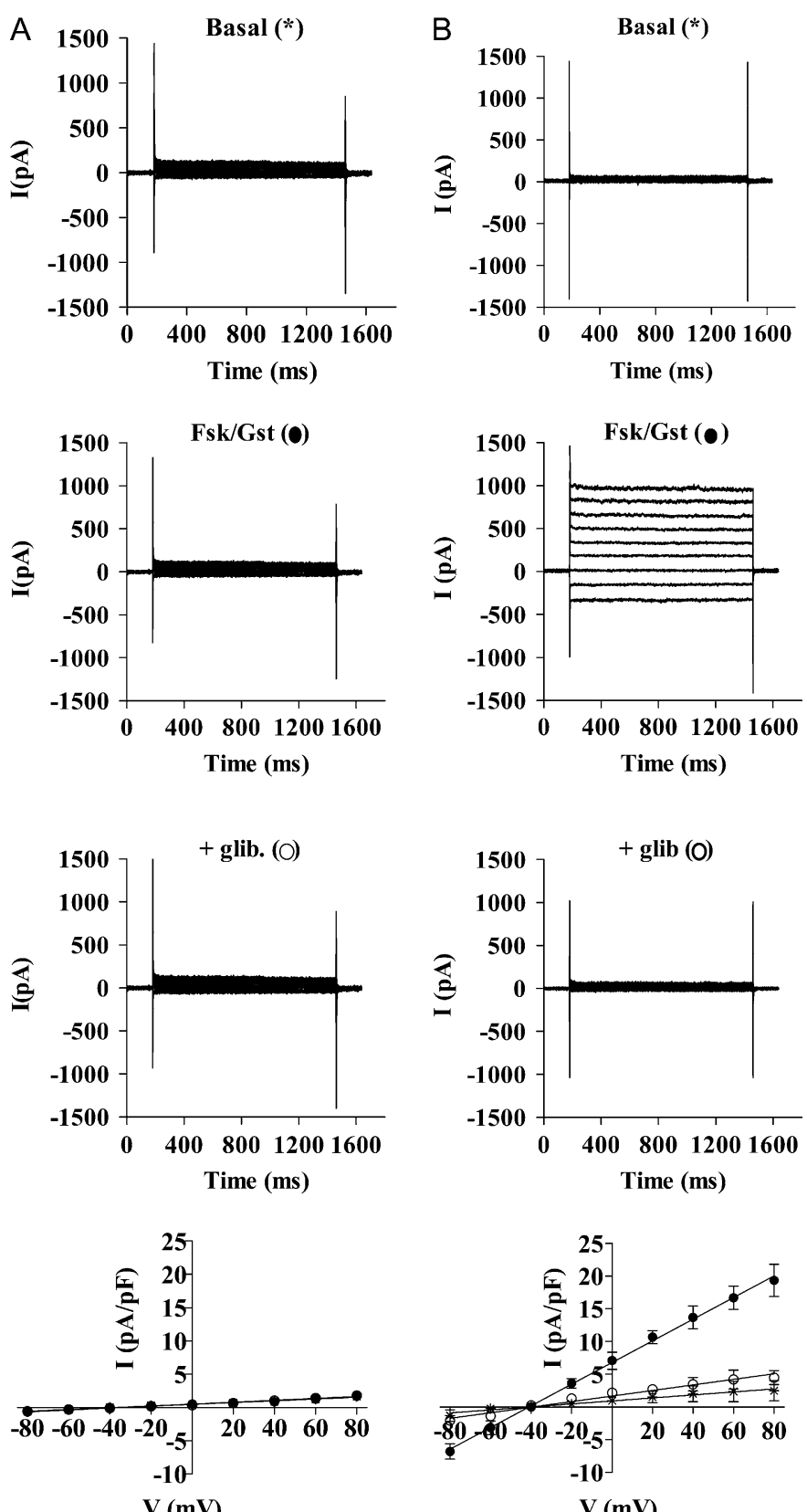

$\mathrm{Ca}^{2+}$ homeostasis, we were able to show parallel normalization of these parameters correlated with the restoration of the F508del-CFTR-dependent secretion. Therefore, we provide here the first evidence that a respiratory CF cell can acquire a nonCF-like phenotype when chronically treated with low concentrations of a pharmacological drug.

Manipulation of aberrant intracellular F508del-CFTR trafficking has great promise as a pharmacological treatment for the majority of patients with CF. This approach, inducing the expression of a functional native protein by correcting its intracellular trafficking, would circumvent many of the problems associated with mutations in the CFTR gene, such as abnormal $\mathrm{NaCl}$ transport. Altered ionic transport causes airway surface liquid volume depletion, delayed mucus clearance, and mucus adhesion to airway surfaces (8). This pathogenetic sequence results in airway mucus obstruction, and sets the stage for chronic airway inflammation and bacterial infection. What we found in the present study is that the daily treatment for 2 months with a low concentration of a candidate corrector results
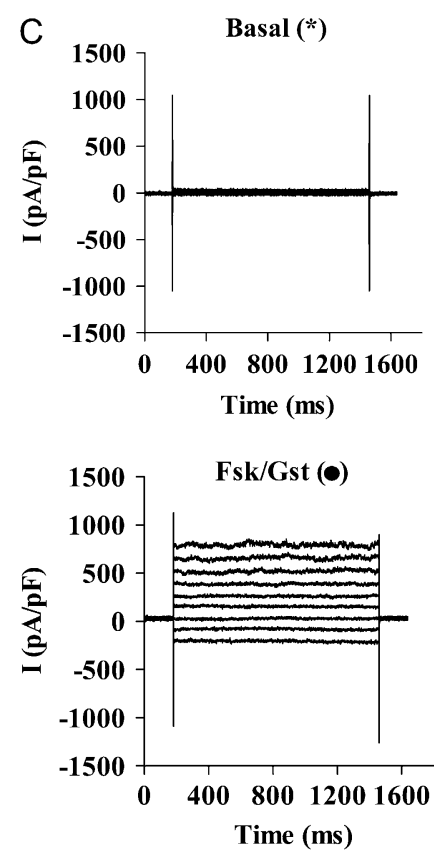

Figure 5. Perforated whole-cell patch-clamp experiments on CF15 cells. Currents were recorded in untreated cells $(A)$ or in miglustatcorrected cells $(B$ and $C)$. In $(B)$, cells were incubated 8 hours with $3 \mu \mathrm{M}$ miglustat. In (C), cells were cultured in the presence of $3 \mu \mathrm{M}$ miglustat for 6 days, with a refreshment of miglustat every 24 hours. For each condition, the example given is the complete sequence of experiments shown for the same cell. The current was recorded in the basal condition (upper traces, asterisk), after addition of $10 \mu \mathrm{M}$ Fsk plus $30 \mu \mathrm{M}$ Gst (Fsk/Gst, middle traces, closed circles), and finally after adding $100 \mu \mathrm{M}$ glibenclamide (glib) or $10 \mu \mathrm{M}$ CFTR $_{\text {inh-172 }}$ (bottom traces, open circles). Corresponding curves for current densities as a function of voltage are presented below each panel $(n=3$ for each condition).

in sustained correction of F508del-CFTR trafficking in phase with a restoration of a CFTR-dependent secretion, but also with a down-regulation of sodium hyperabsorption and regulation of calcium homeostasis. These data should serve as a proof-ofprinciple and as a template to investigate whether a pharmacological agent, able to improve F508del-CFTR function, could be efficient for other cellular dysfunctions attributable to CFTR. During the last decade, the $\mathrm{CF}$ research community has made a great effort to search for potential candidates for future $\mathrm{CF}$ pharmacotherapy. Several interesting small and chemically diverse compounds have been proposed to rescue F508del-CFTR function. This is, for example, the case for curcuminoides, 4PBA (biphenyl), and $\mathrm{CF}_{\text {cor-325 }}(12,15,22,23)$. Indeed, the intracellular trafficking of F508del-CFTR can be experimentally improved by a number of pharmacological agents. The agent 4PBA, although at millimolar concentrations, led to improved F508del-CFTR intracellular trafficking in CF epithelial cells (15), with convincing evidence showing that it regulates the expression of molecular chaperones, such as Heat-shock 
A

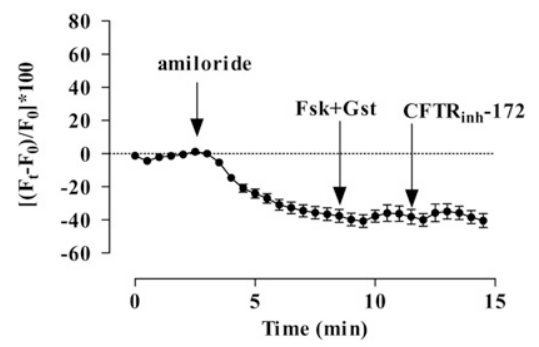

2 days

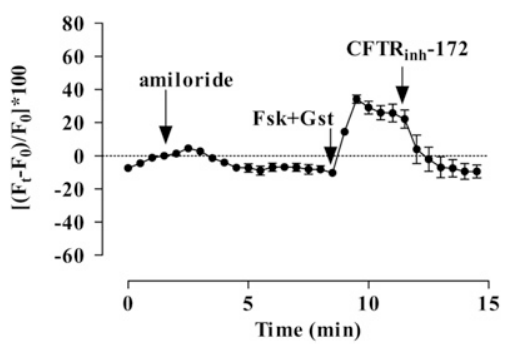

58 days

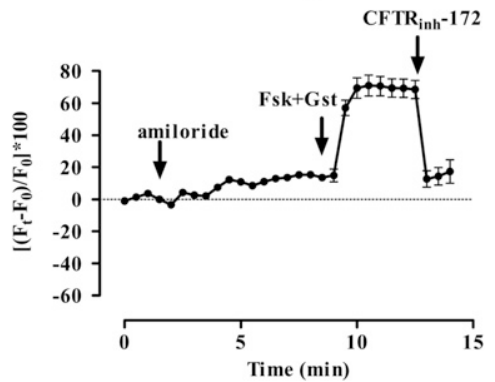

B
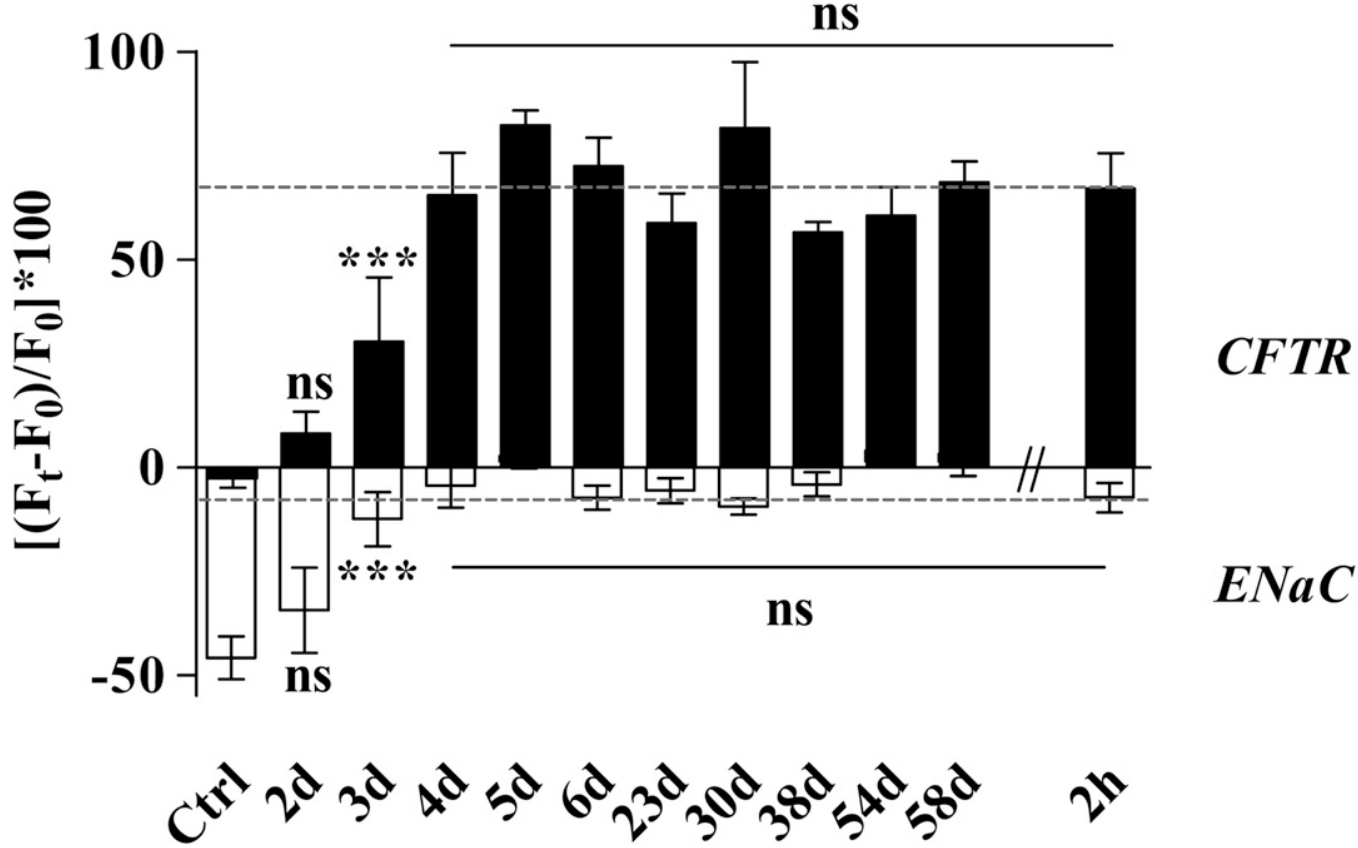

Figure 6. Functional evaluation of F508del-CFTR and epithelial $\mathrm{Na}^{+}$channel (ENaC) by bis-(1,3-diethylthiobarbituric acid)trimethine oxonol (DiSBAC $2[3]$ ) assay in CF15 cells chronically cultured with $3 \mu \mathrm{M}$ miglustat, refreshing miglustat every 24 hours. $(A)$ Examples of typical time courses obtained with untreated cells (Ctrl) or with cells treated for 2 and 58 days. Data represent the mean ( \pm SEM) of the relative fluorescence collected from all the cells of a field (from 12 to 16 ). Amiloride $(100 \mu \mathrm{M})$ is used to inhibit ENaC activity; a mixture of $10 \mu \mathrm{M}$ Fsk plus $30 \mu \mathrm{M}$ Gst is used to activate CFTR. CFTR inh-172 $_{2}(10 \mu \mathrm{M})$ is used to inhibit CFTR. (B) Histograms report the mean of the relative fluorescence collected from separate experiments $(2<N<4)$, with a total of cells from 24 to 48 . "CFTR" corresponds to the Fsk plus Gst-induced fluorescence variation, and "ENaC" to the amiloride-senstive fluorescence variation. The last bar on the right and the dashed line represent the responses obtained on CF15 cells treated with $100 \mu \mathrm{M}$ miglustat for 2 hours. ${ }^{* * *} P<0.001$; ns, not significant.

cognate (Hsc)70 (24) and Heat-shock protein (HSP)70 (25). Enteral administration of 4-PBA to F508del-CFTR mice changed the epithelial ionic transport of the mice, with observation of improved chloride transport without, however, a change in the aberrant sodium transport (22). On the other hand, others have reported that administration of Ca-ATPase pump inhibitors, such as thapsigargin and curcumin, to F508del-CFTR mice normalized both nasal epithelial chloride and sodium transport after rescue of F508del-CFTR trafficking $(12,22)$, an observation also found in airway CF epithelial cells (14). However, high concentrations of curcumin seems to be required to achieve repair of F508del-CFTR trafficking. Although such concentrations are achievable in vivo, it would require patients to take large quantities of the drug.

Most of these small F508del-CFTR correctors act after shortterm incubation (within 24 hours) of CF cells, depending on the studies and laboratories, and little information is available concerning their effects in the long term. In a study identifying the corrector $\mathrm{CF}_{\text {cor-325 }}$ (23), the authors monitored the persistence of correction after washing out the drug, and found that the CFTR-mediated Fsk response could be maintained for up to 36 hours after removal of the compound, and then declined to 72 hours. This compound was identified using a protocol for which cells were preincubated for 16 hours with $10 \mu \mathrm{M}$ of the drug diluted in $0.1 \%$ DMSO (23). For other compounds, F508del-CFTR correction was seen within 3-6 hours, with maximal effect after 12-30 hours, and persistence for more than 12 hours after washout (26). For another study, incubation for 8-10 hours with $1 \mu \mathrm{M}$ thapsigargin selectively rescued trafficking-defective HERG (Human Ether-a-go-go Related Gene) channels (27). In contrast, in an attempt to compare various compounds for their efficacy in promoting maturation of CFTR processing mutants, a recent study showed that thapsigargin, curcumin, and miglustat failed to correct the F508del-CFTR trafficking (17). However, these authors underlined that this opposite result could be the consequence of a difference in the duration of treatment. Indeed, they incubated CF cells with $100 \mu \mathrm{M}$ of miglustat for 24 hours. Our data, highlighting here the importance of both concentration and time of incubation, explain these discrepancies. In partic- 
A

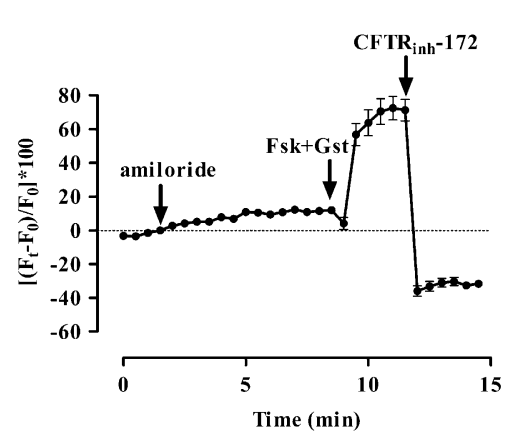

2 days after washout

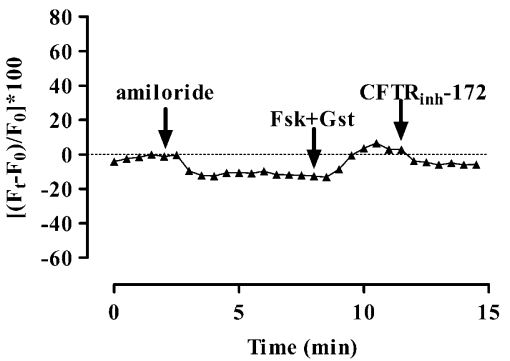

4 days after washout

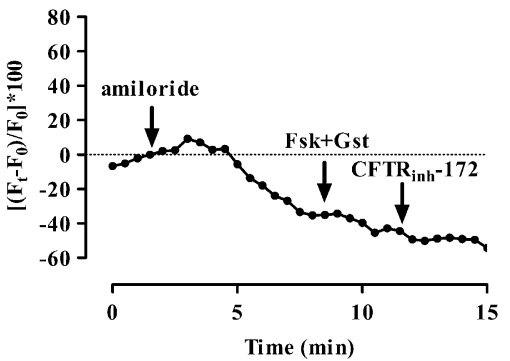

B
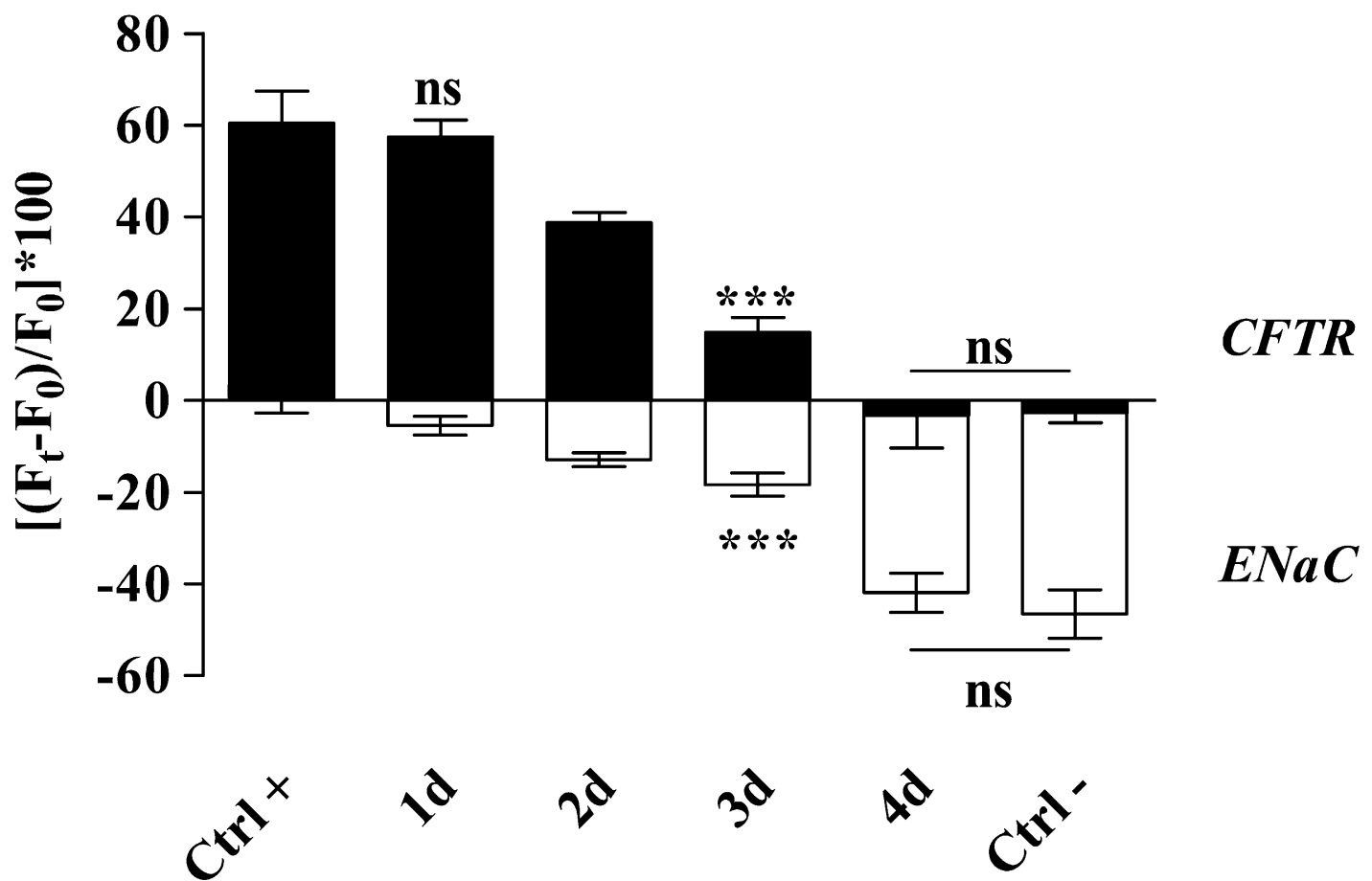

Figure 7. Reversibility of the long-term rescue induced by low-concentration miglustat. CF15 cells were cultured during 54 days with $3 \mu \mathrm{M}$ miglustat, refreshing miglustat every 24 hours, rinsed, and then $\mathrm{ENaC}$ and F508del-CFTR activities were assayed by $\mathrm{DiSBAC}_{2}(3)$ assay at different times after washout. $(A)$ Examples of typical time courses obtained on CF15 cells after 54 days of treatment without washout (left panel), after waiting 2 days (middle panel), or 4 days after washout. Data represent the mean ( \pm SEM) of the relative fluorescence collected from all the cells of a field $(10<\mathrm{n}<12)$. Amiloride $(100 \mu \mathrm{M})$ is used to inhibit ENaC activity; a mixture of $10 \mu \mathrm{M}$ Fsk plus $30 \mu \mathrm{M}$ Gst is used to activate CFTR. CFTR inh-172 $(10 \mu \mathrm{M})$ is used to inhibit CFTR. (B) Histograms report the mean of the relative fluorescence collected from two separate experiments $(n=48$ cells). "CFTR" corresponds to the Fsk plus Gst-induced fluorescence variation, and "ENaC" to the amiloride-senstive fluorescence variation. ${ }^{* \star *} P<0.001$; ns, not significant.

ular, we show here that, after 24 hours, there is no more rescue of F508del-CFTR without renewal of miglustat. It emphasizes the importance of precisely understanding pharmacological parameters of a given drug by exploring more systematically not only concentration, but also time of incubation.

We considered the reversibility of the rescue of F508delCFTR by miglustat as a good indicator in line with the safety of the drug, as reported in the treatment of Gaucher's disease (28). In this regard, even after 2 months of treatment, CF cells kept their ability to return to their basal state (i.e., showing, again, defective $\mathrm{NaCl}$ transport). During chronic treatment, this reversibility occurred within 4 days after washing out the drug. Interestingly, it also took 4 days at the beginning of the treatment to acquire the non-CF-like phenotype, suggesting a potential cumulative effect of the drug for the measurable onset or offset of the rescue process. Further analysis is needed to understand this point.
We showed here a progressive correction of CFTR and $\mathrm{ENaC}$ abnormal functions after repeated incubation of $\mathrm{CF}$ cells with low-concentration $(3 \mu \mathrm{M})$ miglustat. Why is this concentration relevant in the case of human study? A recent pharmacokinetic study reported the profile of miglustat in rat, with a focus on tissues distribution (29). These authors showed that miglustat was well absorbed and exhibited an oral bioavailability of $40-60 \%$. The tissue distribution indicated the presence of miglustat in a number of organs and tissues (in particular, lungs) that are considered of importance for long-term therapeutic benefit. They determined that plasma concentration after oral administration of miglustat $(137 \mathrm{mg} / \mathrm{kg})$ was $12.8( \pm 3.03)$ $\mu \mathrm{g}$ equiv/ml, and that concentration of the drug in many tissues was equal to or exceeded that in plasma (29). For example, they determined a lung concentration of $14.5 \mu \mathrm{g}$ equiv/g, and a small intestine concentration of $294 \mu \mathrm{g}$ equiv/g. More recently, a re- 
view focused on the use of miglustat in adult patients with type 1 Gaucher's disease (30). As miglustat therapy in patients with type 1 Gaucher is $100 \mathrm{mg}$, three times daily, they studied the pharmacokinetic profile of miglustat (100 mg, single dose), and demonstrated a peak plasma concentration of $0.86 \mu \mathrm{g} / \mathrm{ml}(30)$. In our study, we used a $3-\mu \mathrm{M}$ concentration of miglustat, corresponding to $0.77 \mu \mathrm{g} / \mathrm{ml}$. Thus, the concentration of miglustat used in our experiments is comparable to that routinely achieved in the plasma of patients under miglustat therapy for Gaucher's disease. The data on pharmacokinetic properties of miglustat is, however, relatively limited, except for some information at the dosage used in the treatment of type 1 Gaucher's disease. We know that miglustat is rapidly absorbed after oral administration, with a time to maximum plasma concentration of 2.5 hours. The apparent elimination of the compound occurs at 6-7 hours, and the major route of excretion appears to be renal (30). Whether this value can be extrapolated to our model is uncertain, but, to some extent, could explain the short-lived effect of miglustat observed in the first part of our study. Finally, we are not aware of any metabolic derivatives or inactive by-products of miglustat that could contribute to the short-lived effect.

In conclusion, we have shown that a respiratory $\mathrm{CF}$ cell can acquire a non-CF-like phenotype when chronically treated with a low concentration of a pharmacological drug, resulting in progressive, stable, reversible, and sustained correction of F508del-CFTR trafficking, down-regulation of sodium hyperabsorption, and regulation of the calcium homeostasis. This body of information makes the use of miglustat attractive as a potential pharmacologic therapy for those patients with $\mathrm{CF}$ who have at least one F508del-CFTR allele.

Conflict of Interest Statement: None of the authors has a financial relationship with a commercial entity that has an interest in the subject of this manuscript.

Acknowledgment: The authors thank Nathalie Bizard for cell culture and Anne Cantereau for confocal imaging. They are also grateful for the expert technical advice and training of Dr. Giulio Cabrini (Laboratory of Molecular Pathology, Verona, Italy) in ion channel functions by single-cell fluorescence imaging.

\section{References}

1. Sheppard DN, Welsh MJ. Structure and function of the CFTR chloride channel. Physiol Rev 1999;79:S23-S45.

2. Kopito RR. Biosynthesis and degradation of CFTR. Physiol Rev 1999; 79:S167-S173.

3. Heda GD, Tanwani M, Marino CR. The delta F508 mutation shortens the biochemical half-life of plasma membrane CFTR in polarized epithelial cells. Am J Physiol Cell Physiol 2001;280:C166-C174.

4. Dalemans W, Barbry P, Champigny G, Jallat S, Dott K, Dreyer D, Crystal RG, Pavirani A, Lecocq JP, Lazdunski M. Altered chloride ion channel kinetics associated with the delta F508 cystic fibrosis mutation. Nature 1991;354:526-528.

5. Kunzelmann K, Mall M. Pharmacotherapy of the ion transport defect in cystic fibrosis. Clin Exp Pharmacol Physiol 2001;28:857-867.

6. Mall M, Kunzelmann K. Correction of the CF defect by curcumin: hypes and disappointments. Bioessays 2005;27:9-13.

7. Zeitlin PL. Future pharmacological treatment of cystic fibrosis. Respiration 2000;67:351-357.

8. Boucher RC. Cystic fibrosis: a disease of vulnerability to airway surface dehydration. Trends Mol Med 2007;13:231-240.

9. Norez C, Noel S, Wilke M, Bijvelds M, Jorna H, Melin P, DeJonge H, Becq F. Rescue of functional delF508-CFTR channels in cystic fibrosis epithelial cells by the alpha-glucosidase inhibitor miglustat. FEBS Lett 2006;580:2081-2086.

10. Antigny F, Norez C, Becq F, Vandebrouck C. Calcium homeostasis is abnormal in cystic fibrosis airway epithelial cells but is normalized after rescue of F508del-CFTR. Cell Calcium 2008;43:175-183.

11. Noel S, Wilke M, Bot AG, De Jonge HR, Becq F. Parallel improvement of sodium and chloride transport defects by miglustat (n- butyldeoxynojyrimicin) in cystic fibrosis epithelial cells. J Pharmacol Exp Ther 2008;325:1016-1023.

12. Egan ME, Glockner-Pagel J, Ambrose C, Cahill PA, Pappoe L, Balamuth N, Cho E, Canny S, Wagner CA, Geibel J, et al. Calciumpump inhibitors induce functional surface expression of delta F508CFTR protein in cystic fibrosis epithelial cells. Nat Med 2002;8:485-492.

13. Lipecka J, Norez C, Bensalem N, Baudouin-Legros M, Planelles G, Becq F, Edelman A, Davezac N. Rescue of deltaF508-CFTR (cystic fibrosis transmembrane conductance regulator) by curcumin: involvement of the keratin 18 network. J Pharmacol Exp Ther 2006;317:500-505.

14. Norez C, Antigny F, Becq F, Vandebrouck C. Maintaining low $\mathrm{Ca}^{2+}$ level in the endoplasmic reticulum restores abnormal endogenous F508delCFTR trafficking in airway epithelial cells. Traffic 2006;7:562-573.

15. Rubenstein RC, Egan ME, Zeitlin PL. In vitro pharmacologic restoration of CFTR-mediated chloride transport with sodium 4-phenylbutyrate in cystic fibrosis epithelial cells containing delta F508-CFTR. J Clin Invest 1997;100:2457-2465.

16. Rubenstein RC, Zeitlin PL. A pilot clinical trial of oral sodium 4phenylbutyrate (buphenyl) in deltaF508-homozygous cystic fibrosis patients: partial restoration of nasal epithelial CFTR function. Am J Respir Crit Care Med 1998;157:484-490.

17. Wang Y, Loo TW, Bartlett MC, Clarke DM. Modulating the folding of $\mathrm{P}$-glycoprotein and cystic fibrosis transmembrane conductance regulator truncation mutants with pharmacological chaperones. Mol Pharmacol 2007;71:751-758.

18. Jefferson DM, Valentich JD, Marini FC, Grubman SA, Iannuzzi MC, Dorkin HL, Li M, Klinger KW, Welsh MJ. Expression of normal and cystic fibrosis phenotypes by continuous airway epithelial cell lines. Am J Physiol 1990;259:L496-L505.

19. Norez C, Heda GD, Jensen T, Kogan I, Hughes LK, Auzanneau C, Derand R, Bulteau-Pignoux L, Li C, Ramjeesingh M, et al. Determination of CFTR chloride channel activity and pharmacology using radiotracer flux methods. J Cyst Fibros 2004;3:119-121.

20. Norez C, Pasetto M, Dechecchi MC, Barison E, Anselmi C, Tamanini A, Quiri F, Cattel L, Rizzotti P, Dosio F, et al. Chemical conjugation of \{Delta\}F508-CFTR corrector deoxyspergualin to transporter human serum albumin enhances its ability to rescue CI channel functions. Am J Physiol Lung Cell Mol Physiol 2008.295:L336-L347.

21. Dall'Asta V, Gatti R, Orlandini G, Rossi PA, Rotoli BM, Sala R, Bussolati O, Gazzola GC. Membrane potential changes visualized in complete growth media through confocal laser scanning microscopy of bis-oxonol-loaded cells. Exp Cell Res 1997;231:260-268.

22. Egan ME, Pearson M, Weiner SA, Rajendran V, Rubin D, GlocknerPagel J, Canny S, Du K, Lukacs GL, Caplan MJ. Curcumin, a major constituent of turmeric, corrects cystic fibrosis defects. Science 2004; 304:600-602.

23. Van Goor F, Straley KS, Cao D, Gonzalez J, Hadida S, Hazlewood A, Joubran J, Knapp T, Makings LR, Miller M, et al. Rescue of deltaF508-CFTR trafficking and gating in human cystic fibrosis airway primary cultures by small molecules. Am J Physiol Lung Cell Mol Physiol 2006;290:L1117-L1130.

24. Rubenstein RC, Zeitlin PL. Sodium 4-phenylbutyrate downregulates Hsc70: implications for intracellular trafficking of deltaF508-CFTR. Am J Physiol Cell Physiol 2000;278:C259-C267.

25. Choo-Kang LR, Zeitlin PL. Induction of HSP70 promotes deltaF508 CFTR trafficking. Am J Physiol Lung Cell Mol Physiol 2001;281: L58-L68.

26. Pedemonte N, Lukacs GL, Du K, Caci E, Zegarra-Moran O, Galietta LJ, Verkman AS. Small-molecule correctors of defective deltaF508CFTR cellular processing identified by high-throughput screening. J Clin Invest 2005;115:2564-2571.

27. Delisle BP, Anderson CL, Balijepalli RC, Anson BD, Kamp TJ, January CT. Thapsigargin selectively rescues the trafficking defective LQT2 channels G601S and F805C. J Biol Chem 2003;278:35749-35754.

28. Giraldo P, Latre P, Alfonso P, Acedo A, Alonso D, Barez A, Corrales A, Franco R, Roldan V, Serrano S, et al. Short-term effect of miglustat in every day clinical use in treatment-naive or previously treated patients with type 1 Gaucher's disease. Haematologica 2006;91:703-706.

29. Treiber A, Morand O, Clozel M. The pharmacokinetics and tissue distribution of the glucosylceramide synthase inhibitor miglustat in the rat. Xenobiotica 2007;37:298-314.

30. McCormack PL, Goa KL. Miglustat. Drugs 2003;63:2427-2434. 